\title{
Advances in Large-area Sample Preparation Using Broad Argon Ion Beam Milling for Multiphase Materials
}

Pawel Nowakowski, Mary Ray and Paul Fischione

\section{E.A. Fischione Instruments Inc., Export, Pennsylvania, United States}

One of the more challenging tasks in materials science is accurate sample preparation for multiple application and characterization techniques. In the last decade, an emerging sample preparation technique for a variety of microscopy and microanalyses applications was argon broad ion beam milling (BIB) [15]. BIB milling can have some limitations, however. The most notable limitation is the relatively small milling area, which ranges from hundreds of micrometers up to $5 \mathrm{~mm}$ diameter. This limitation prevents application of BIB technology where a large field of observation ( $25 \mathrm{~mm}$ diameter or larger) is required to fully understand material structure and properties. While a larger sample can be divided into smaller pieces to accommodate BIB maximum milling areas, this often introduces defects (such as strain, cracks, or delamination) to the sample. Defects induced by cutting materials into smaller sample sizes, as well as defects induced by alternate sample preparation methods (such as mechanical preparation) can alter the crystalline structure of the sample by strain induction $[6,7]$ and render attempts to accurately characterize material structure and properties impossible. Multiphase materials present additional issues; because different phases can have very different physicochemical characteristics. For example, if one phase is hard and brittle and the second phase is soft and ductile, the rate of material removal during mechanical polishing or electropolishing can vary widely. In addition, the softer phase will be more affected by plastic strain introduction during polishing. For these reasons, is generally accepted that ion milling is the preferred method of sample preparation for multiphase materials.

The paper describes how a recently developed low-energy, BIB milling instrument can improve and facilitate large area sample preparation. The advantages of this instrumentation include effective milling of an area up to $50 \mathrm{~mm}$ in diameter and user-adjustable argon ion beam parameters (energy, spot size, current density, and position), which offer precise control of the milling process.

The following investigations into large-area sample preparation are discussed:

- multiphase materials in a large geological sample [Figures 1],

- microstructure of a large weld section, and

- cross section of a large microelectronics device [Figure 2].

Additional studies of large-area milling of multiphase materials are underway. 

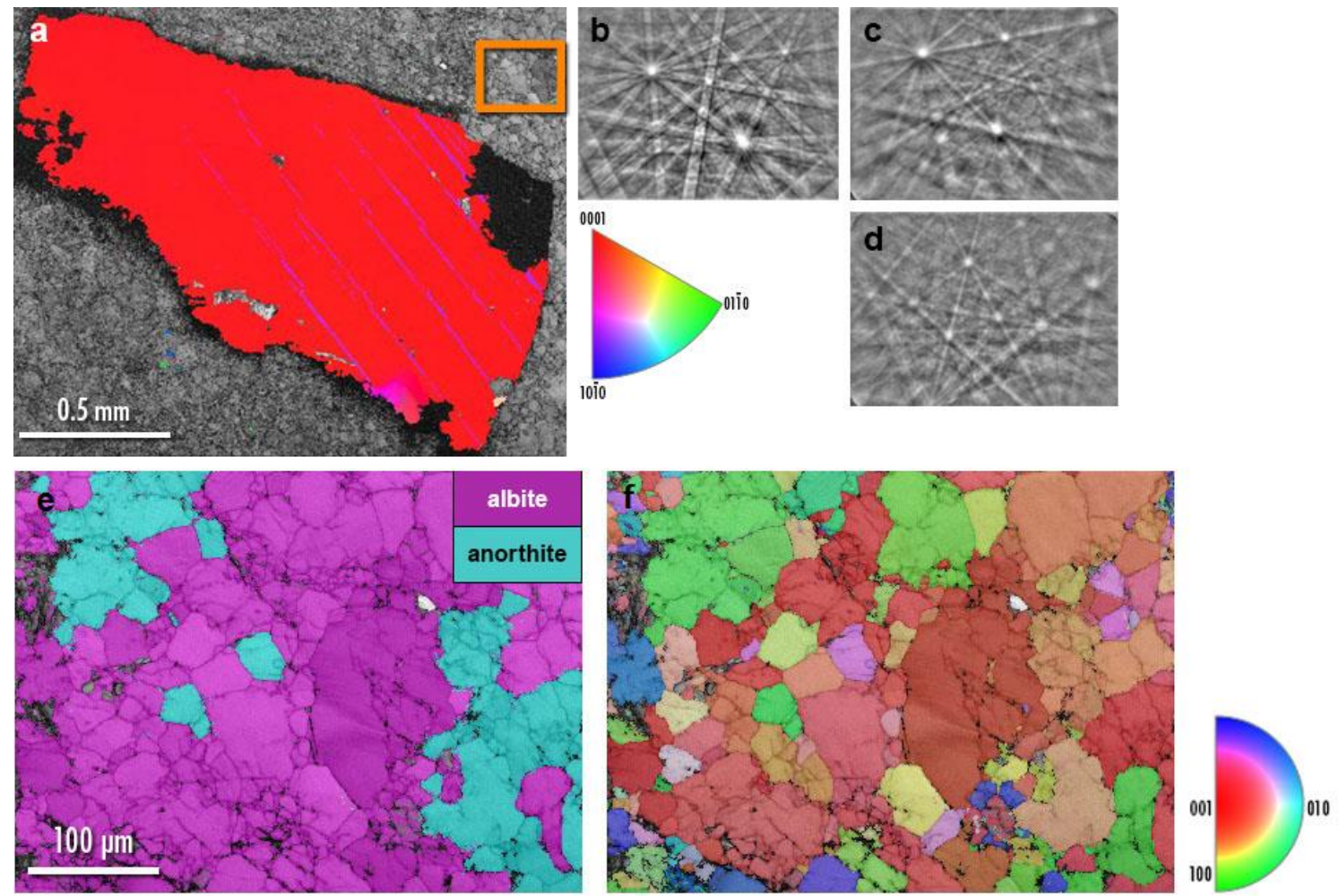

Figure 1. A segment of a large geological sample (a) showing a large grain of alumina (inverse pole figure [IPF] electron backscatter diffraction [EBSD] map), surrounded by smaller grains of different minerals identified as primarily albite and anorthite (presented as a band contrast EBSD map). Map collected at 20 $\mathrm{kV}$ using $5 \mu \mathrm{m}$ step size. EBSD patterns of alumina (b), anorthite (c), and albite (d). EBSD phase distribution map (e) and IPF map (f) collected from the area marked by the yellow rectangle on Figure 1a. Maps collected at $20 \mathrm{kV}$ using $1 \mu \mathrm{m}$ step size.
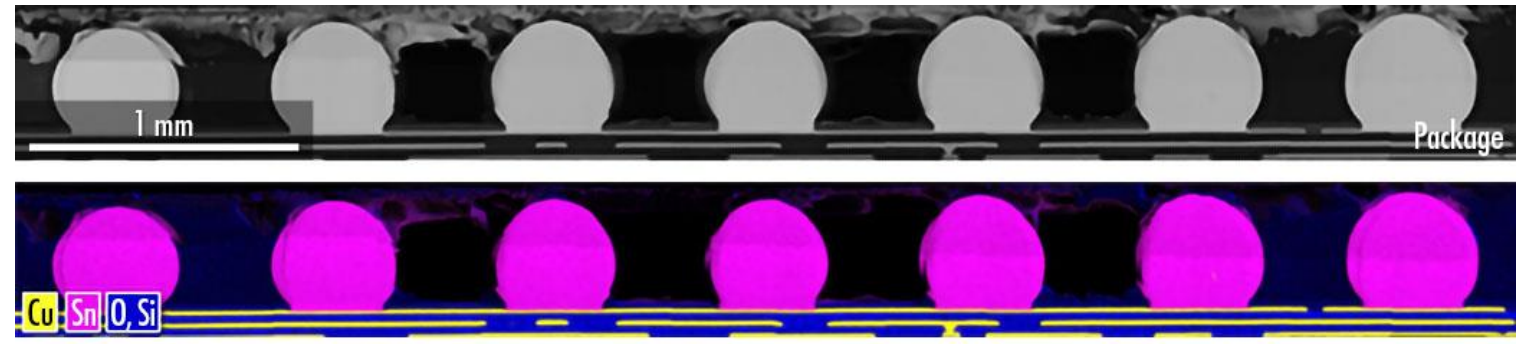

Figure 2. Large cross-section sample from a microelectronics chip package. Backscattered electron contrast image of solder bumps (top), and energy-dispersive X-ray spectroscopy overlay maps of $\mathrm{Cu}, \mathrm{Sn}$, $\mathrm{O}$, and $\mathrm{Si}$ (bottom).

\section{References}

1. Nowakowski, P., Wiezorek, J., Spinelli, I., Ray, M., \& Fischione, P. (2019). Elastic and plastic strain measurement using electron backscatter diffraction technique: The influence of sample preparation. Microscopy and Microanalysis, 25(S2), 534-535.

2. Nowakowski, P., Bonifacio, C., Ray, M., \& Fischione, P. (2019). Combining emerging sample preparation methods, SEM, and TEM investigations for microelectronics device characterization at multiple scales. Microscopy and Microanalysis, 25(S2), 688-689. 
3. Nowakowski, P., Wiezorek, J., Bathula, V., Mielo, S., Khanal, S., Bonifacio, C., \& Fischione, P. (2018). SEM and TEM characterization of plastic deformation structures in aluminum by EBSD, TKD, and PEDbased orientation imaging techniques. Microscopy and Microanalysis, 24(S1), 2182-2183.

4. Nowakowski, P., Bonifacio, C. S., Campin, M. J., Ray, M. L., Fischione, P. E., \& Mathieu, S. (2017). EBSD and TEM microstructural studies of new fuel cladding in generation IV sodium-cooled fast nuclear reactors. Microscopy and Microanalysis, 23(S1), 2224-2225.

5. Nowakowski, P., Schlenker, J., Ray, M., \& Fischione, P. (2016). Sample preparation using broad argon ion beam milling for electron backscatter diffraction (EBSD) analysis. Microscopy and Microanalysis, 22(S3), 12-13.

6. I. Wright, M.M. Nowell, D.P. Field, (2011). A Review of Strain Analysis Using Electron Backscatter Diffraction Microscopy and Microanalysis, 17, 316-329.

7. Katrakova, F. Mucklich, (2002). Specimen preparation for electron backscatter diffraction - Part II: Ceramics, Pract. Metallog. 39, 644-662 